\title{
The effect of local anaesthetic wound infiltration on chronic pain after lower limb joint replacement: A protocol for a double-blind randomised controlled trial
}

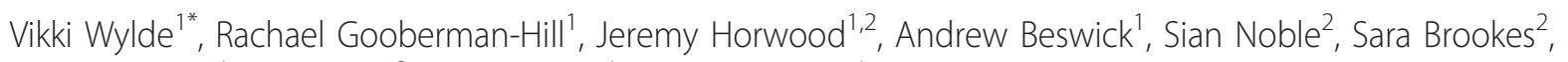
Alison J Smith', Mark Pyke ${ }^{3}$, Paul Dieppe ${ }^{4}$, Ashley W Blom

\begin{abstract}
Background: For the majority of patients with osteoarthritis $(\mathrm{OA})$, joint replacement is a successful intervention for relieving chronic joint pain. However, between 10-30\% of patients continue to experience chronic pain after joint replacement. Evidence suggests that a risk factor for chronic pain after joint replacement is the severity of acute post-operative pain. The aim of this randomised controlled trial (RCT) is to determine if intra-operative local anaesthethic wound infiltration additional to a standard anaethesia regimen can reduce the severity of joint pain at 12-months after total knee replacement (TKR) and total hip replacement (THR) for OA.
\end{abstract}

Methods: 300 TKR patients and 300 THR patients are being recruited into this single-centre double-blind RCT. Participants are recruited before surgery and randomised to either the standard care group or the intervention group. Participants and outcome assessors are blind to treatment allocation throughout the study. The intervention consists of an intra-operative local anaesthetic wound infiltration, consisting of 60 $\mathrm{mls}$ of $0.25 \%$ bupivacaine with 1 in 200,000 adrenaline. Participants are assessed on the first 5 days postoperative, and then at 3-months, 6-months and 12-months. The primary outcome is the WOMAC Pain Scale, a validated measure of joint pain at 12-months. Secondary outcomes include pain severity during the inpatient stay, post-operative nausea and vomiting, satisfaction with pain relief, length of hospital stay, joint pain and disability, pain sensitivity, complications and cost-effectiveness. A nested qualitative study within the RCT will examine the acceptability and feasibility of the intervention for both patients and healthcare professionals.

Discussion: Large-scale RCTs assessing the effectiveness of a surgical intervention are uncommon, particulary in orthopaedics. The results from this trial will inform evidence-based recommendations for both short-term and long-term pain management after lower limb joint replacement. If a local anaesthetic wound infiltration is found to be an effective and cost-effective intervention, implementation into clinical practice could improve long-term pain outcomes for patients undergoing lower limb joint replacement.

Trial registration: Current Controlled Trials ISRCTN96095682

\footnotetext{
* Correspondence: V.Wylde@bristol.ac.uk

${ }^{1}$ Musculoskeletal Research Unit, School of Clinical Sciences, University of

Bristol, Avon Orthopaedic Centre, Southmead Hospital, Bristol, BS10 5NB, UK

Full list of author information is available at the end of the article
}

(C) 2011 Wylde et al; licensee BioMed Central Ltd. This is an Open Access article distributed under the terms of the Creative Commons 


\section{Background}

Joint replacement is one of the most common elective surgical procedures, with over 150,000 total hip replacements (THR) and total knee replacements (TKR) performed annually in England and Wales in the National Health Service (NHS) [1]. Although chronic pain is the main indication for joint replacement, it is also a common occurrence after surgery. Studies have estimated the incidence of chronic pain after joint replacement to be in the region of $10-30 \%$ [2-4]. Chronic pain after surgery is defined by the International Association for the Study of Pain (IASP) as pain that develops after a surgical intervention and is of at least 3-months duration [5]. Chronic pain is a widely-observed phenomenon after many surgical procedures including breast surgery [6], inguinal hernia repair [7] and thoracic surgery [8]. Research evidence suggests that an important risk factor for chronic pain after surgery is the severity of acute post-operative pain $[9,10]$. Therefore, in addition to causing unnecessary distress and discomfort during the post-operative period, poorly managed post-operative pain can have negative consequences for long-term surgical outcomes. Adequate management of post-operative pain still poses a significant challenge to healthcare, as demonstrated by a review of the literature which concluded that $30 \%$ of patients experience moderate or severe pain after surgery [11]. In a recent study at the Avon Orthopaedic Centre, 58\% of TKR patients and $47 \%$ of THR patients reported moderate or severe pain on the first post-operative day [12]. Therefore, there is a need for trials of interventions targeting acute postoperative pain with the ultimate aim of improving longterm outcomes after joint replacement.

In the context of joint replacement, post-operative pain management needs to provide effective pain relief, while allowing for early mobilisation and rehabilitation [13]. Although many of the traditional methods of achieving peri-operative pain relief can hinder early mobilisation [14], a review of the use of multimodal analgesia in different conditions found that a combination of systemic opioids, nonsteroidal anti-inflammatory drugs, and epidural analgesics or wound infiltration can provide effective pain control and improve post-operative recovery [15]. In joint replacement, there is evidence that multimodal analgesia can give good pain relief with minimal side effects $[16,17]$. A systematic review of a wide range of surgical procedures concluded that the use of local anaesthetic wound infiltration as part of a multimodal approach reduced post-operative pain, opioid use, nausea and vomiting, improved patient satisfaction and reduced the length of hospital stay [18]. Fifteen randomised controlled trials (RCT) in joint replacement identified up to August 2010 are described in Table 1 [19-33]. The results from these studies found that short-term outcomes after joint replacement using multimodal analgesia were broadly favourable. However, with only one small study reporting outcomes after six months, there is little evidence on longer-term pain outcome.

The existing literature provides evidence that the use of local anaesthetic wound infiltration as part of a multimodal anaesthetic regimen reduces short-term postoperative pain after joint replacement. However, it is not known if the reduction of this short-term postoperative pain reduces the severity of chronic pain. The aim of this RCT is to determine if using intra-operative local anaesthetic wound infiltration significantly reduces the severity of joint pain at 12-months after primary TKR or THR.

\section{Methods}

The Arthroplasty Pain Experience (APEX) study is a single-centre double-blind RCT that is currently being conducted at the Avon Orthopaedic Centre, one of the largest orthopaedic centres in the UK. The study has been approved by Southampton and South West Hampshire Research Ethics Committee (B) (09/H0504/94) and all participants provide informed, written consent. The trial has been registered with EudraCT (2009-01381793) and Current Controlled Trials (ISRCTN96095682). The trial is also registered as a Clinical Trial of a Investigational Medicinal Produce with the Medicine Healthcare and Regulary Authority (18524/0215/001-0001).

\section{Study duration}

Recruitment into the trial began in November 2009 and 12-month follow-up for all participants is anticipated to be complete by November 2012 .

\section{Participant recruitment}

Potential participants are screened and recruited by research nurses from pre-operative assessment clinics at the Avon Orthopaedic Centre. Inclusion criteria include being listed for a primary unilateral TKR or THR for osteoarthritis (OA) and being willing and able to provide fully-informed consent. Exclusion criteria include i.) any medical co-morbidity that precludes spinal anaesthesia, regional blocks or the use of strong analgesics postoperatively ii.) severe dementia or psychiatric illness such that they are unable to complete the questionnaires or provide informed consent iii.) listed for simultaneous bilateral joint replacement iv.) having been in the APEX trial for a previous joint replacement v.) being unable to understand English as not all the questionnaires have been translated and validated into other languages. In order to explore generalisability (the patients enrolled in the study being representative of those undergoing joint replacement) anonymised data about age and gender is recorded for all eligible patients. 
Table 1 Randomised controlled trials of local anaesthetic wound infiltration in hip or knee joint replacement (Continued)

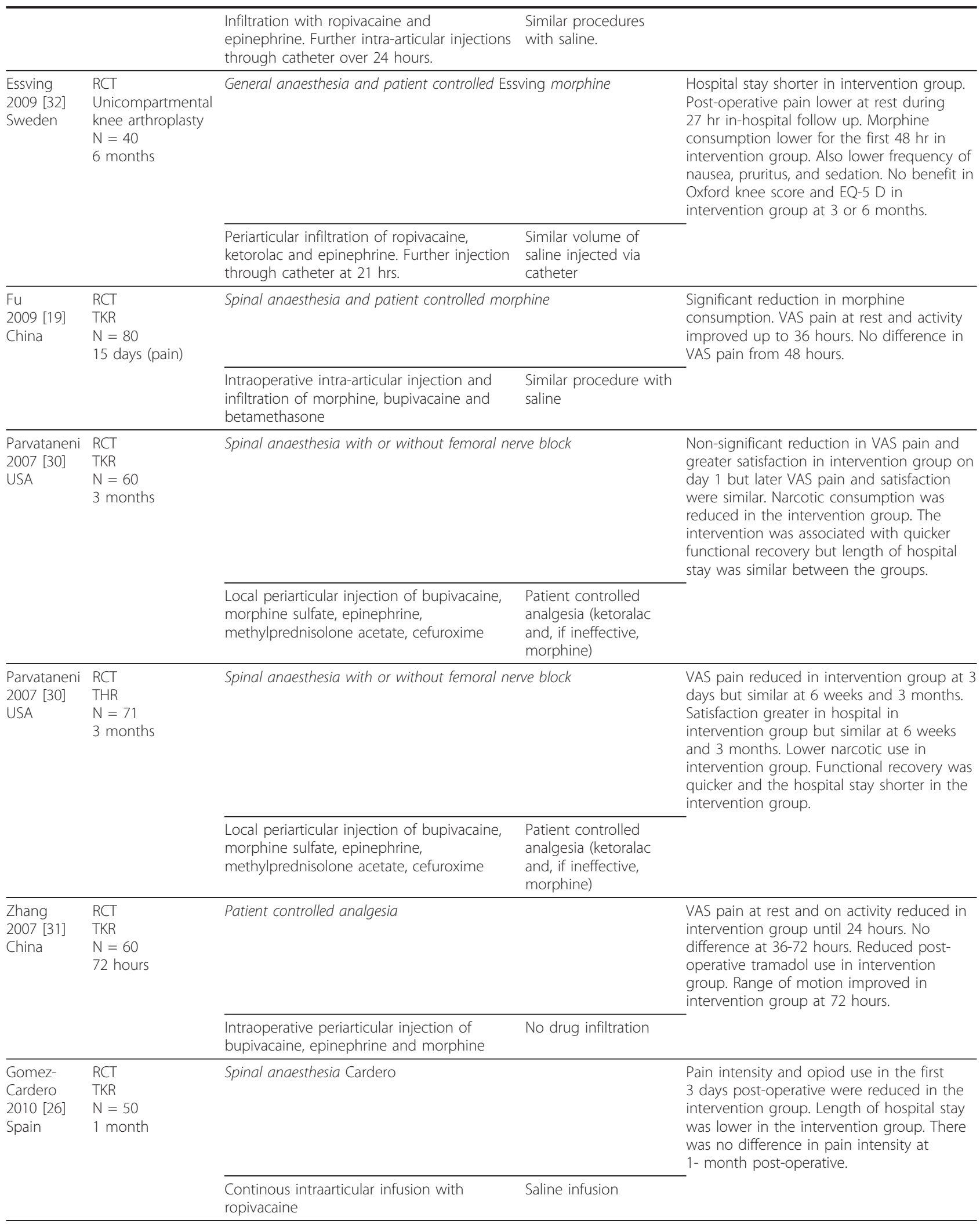


Table 1 Randomised controlled trials of local anaesthetic wound infiltration in hip or knee joint replacement (Continued)

\begin{tabular}{|c|c|c|c|c|}
\hline \multirow{2}{*}{$\begin{array}{l}\text { Essving } \\
2010[27] \\
\text { Sweden }\end{array}$} & \multirow{2}{*}{$\begin{array}{l}\text { RCT } \\
\text { TKR } \\
\mathrm{N}=48 \\
3 \text { months }\end{array}$} & \multicolumn{2}{|c|}{ General anaesthesia and patient controlled morphine } & \multirow{2}{*}{$\begin{array}{l}\text { Morphine consumption and pain intensity } \\
\text { on movement was reduced in the } \\
\text { intervention group in the first } 48 \text { hours } \\
\text { post-operative. Patient satisfaction was } \\
\text { higher in the intervention group. Length of } \\
\text { stay was not affected and no significant } \\
\text { benefit was evident at 3-months post- } \\
\text { operative. }\end{array}$} \\
\hline & & $\begin{array}{l}\text { Intraarticular injection of ropivacaine, } \\
\text { ketorolac and epinephrine during surgery } \\
\text { and through a catheter at } 21 \text { hours post- } \\
\text { operatively }\end{array}$ & $\begin{array}{l}\text { No injection during } \\
\text { surgery and saline } \\
\text { injection at } 21 \text { hours } \\
\text { post-operatively }\end{array}$ & \\
\hline \multirow[t]{2}{*}{$\begin{array}{l}\text { Chen } \\
2010 \text { [28] } \\
\text { Taiwan }\end{array}$} & $\begin{array}{l}\mathrm{RCT} \\
\mathrm{THR} \\
\mathrm{N}=92 \\
\text { Discharge }\end{array}$ & General anaesthesia & & \multirow[t]{2}{*}{$\begin{array}{l}\text { Intervention group had a longer mean time } \\
\text { to first narcotic rescue, but there was no } \\
\text { difference in pain relief, narcotic use or } \\
\text { length of stay. }\end{array}$} \\
\hline & & $\begin{array}{l}\text { Continous intra-articular infusion of } \\
\text { bupivacaine via a infusion pump for } 48 \\
\text { hours }\end{array}$ & $\begin{array}{l}\text { Continous intra- } \\
\text { articular infusion of } \\
\text { saline via a infusion } \\
\text { pump for } 48 \text { hours }\end{array}$ & \\
\hline
\end{tabular}

$\mathrm{RCT}=$ randomised controlled trial, $\mathrm{TKR}=$ total knee replacement, $\mathrm{THR}=$ total hip replacement, $\mathrm{TJR}=$ total joint replacement.

\section{Randomisation}

Before participants undergo surgery, they are randomised to either the standard care group or the intervention group. Randomisation is conducted by means of a computer-generated code, administered centrally and communicated via the internet (through the Bristol Randomised Trials Collaboration). Randomisation is stratified by operation site (TKR/THR) and minimised by baseline joint pain severity and surgical approach. The operating surgeon and anaesthetist are blind to the results of randomisation until the beginning of the operation, when they are informed of treatment allocation by a member of the research team who is not involved in outcomes assessment. The study participants, research nurses involved in recruitment and assessment, and clinical staff involved in the care of study participants are blind to treatment allocation throughout the study.

\section{Intervention}

\section{Hip replacement participants}

Standard care group The standard anaesthetic care for THR participants is a spinal anaesthetic with $3 \mathrm{mls}$ of $0.5 \%$ plain bupivacaine placed at the L3,4 or L4,5 interspace. Intra-operatively, the patient is either awake, sedated or under a light general anaesthetic, depending on patient and anaesthetic factors. If there is intraoperative discomfort then rescue analgesia in the form of intravenous fentanyl is titrated to effect. All participants are given $1 \mathrm{~g}$ of intravenous paracetamol $30 \mathrm{~min}$ utes before the end of the operation. In the recovery area immediately post-operative, participants receive (if no contra-indications are present) $400 \mathrm{mg}$ of ibuprofen administered orally. A patient controlled analgesia (PCA) device is started containing morphine $1 \mathrm{mg} / \mathrm{ml}$, a $1 \mathrm{mg}$ bolus dose and a 5 minute lock-out. If on awakening the patient is in pain with a rating of more than $50 \mathrm{~mm}$ on a $100 \mathrm{~mm}$ pain visual analogue scale (VAS), a morphine bolus up to $0.2 \mathrm{mg} / \mathrm{kg}$ can be administered as rescue analgesia.

Each day during their hospital stay, participants receive a visit from a pain specialist nurse. Post-operative analgesia consists of oral or intravenous paracetamol every 6 hours and, if no contraindications are present, oral ibuprofen $400 \mathrm{mg}$ every 8 hours. When the PCA is no longer needed, oral codeine phosphate 30-60 mg every 6 hours, tramadol 50-100 mg every 6 hours and oramorph 10-20 mg are prescribed as rescue analgesia.

Intervention group The intervention group receives the same anaesthetic and analgesic regime as the standard care group, plus an intra-operative local anaesthetic wound infiltration. The local anaesthetic mixture consists of $60 \mathrm{mls}$ of $0.25 \%$ bupivacaine with 1 in 200,000 adrenaline. If the patient is below $60 \mathrm{~kg}$ or particularly frail, the volume of injectate is reduced to $50 \mathrm{mls}$ or lower if necessary. The surgeon injects the anaesthetic mixture into the joint capsule and short external rotators, fascia, fat and subcutaneous tissue.

Knee replacement participants

Standard care group The standard anaesthetic care for TKR participants is a femoral nerve block and a spinal or general anaesthetic, depending on patient factors. The femoral nerve blockade is achieved by an injection of $20 \mathrm{mls}$ of $0.25 \%$ bupivacaine sited with the use of a nerve stimulator and/or ultrasound guidance. Intra-operative analgesia is provided by titration of intravenous fentanyl 
initially and morphine if deemed necessary in order to achieve haemodynamic stability and a smooth emergence. All participants are given $1 \mathrm{~g}$ of intravenous paracetamol 30 minutes before the end of the operation. The post-operative pain management regime for TKR participants is the same as that described for THR participants. Intervention group The intervention group receive the same anaesthetic and analgesic regime as the standard care group, plus an intra-operative local anaesthetic wound infiltration. The local anaesthetic mixture is the same as described for THR participants. The surgeon injects the anaesthetic mixture into the posterior capsule, medial and lateral capsule, fascia and muscle, and subcutaneous tissues.

\section{Assessment times}

Participants are followed-up for 12-months after surgery. Assessments are conducted pre-operatively, daily during the hopsital stay, and then at 3-months, 6months and 12-months post-operative. Outcomes are assessed using self-report questionnaires, joint examinations, analysis of $\mathrm{x}$-rays, pressure algometry and extraction of data from hospital records.

\section{Primary outcome measure}

The primary outcome is the 12-month Western Ontario and McMaster Universities Index of Osteoarthritis (WOMAC) Pain Scale [34]. The WOMAC Pain score is a widely-used and validated questionnaire to assess the severity of joint pain in OA patients [35]. The severity of joint pain is assessed when performing 5 different activities: walking, using stairs, sitting or lying, standing upright and in bed. Response options for each item are on a 5-point ordered response scale, ranging from none to extreme, which are summed and then transformed into a 0-100 scale (extreme pain-no pain).

\section{Secondary outcome measures}

\section{Pain severity during the in-patient stay}

On the day of surgery, participants are asked to verbally rate their pain severity on a $0-10$ scale when they wake up in the recovery room and when they leave recovery, and then on a 4-hourly basis when they are transfeered to a ward. From the day after surgery to discharge (or up to post-operative day 5), participants complete a $100 \mathrm{~mm}$ VAS for pain severity at rest and $100 \mathrm{~mm}$ VAS for pain severity on movement in the morning, afternoon and evening.

\section{Satisfaction with in-patient pain relief}

At the end of each day, participants complete a $100 \mathrm{~mm}$ VAS to indicate their satisfaction with their pain relief. Nausea and vomiting during the in-patient stay Participants are asked whether they have experienced any vomiting or nausea (yes/no variable) each day. If participants have experienced nausea, they then indicate how distressing it was on a $100 \mathrm{~mm}$ VAS (not at all distressing to very distressing).

\section{Length of hospital stay}

This is calculated from participants admission and discharge dates.

\section{Joint pain and disability}

The WOMAC [34] and the Intermittent and Constant Osteoarthritis Pain (ICOAP) [36] are completed pre-operatively, and then at 3-months, 6-months and 12-months post-operatively. To assess whether the intervention reduces the incidence of chronic neuropathic pain, participants complete the PainDETECTquestionnaire [37] at 12-months post-operative.

\section{Pressure algometry}

Pressure pain thresholds, which are a measure of pain sensitivity, are measured on the volar forearm before surgery, on discharge from hospital, and 12-month postoperative using a pressure algometer (Somedic, Sweden).

\section{Medical and surgical complications}

This data is recorded from hospital records during the in-patient stay, a telephone call to participants at 3months post-operative, and a comprehensive joint assessment by a research nurse and an x-ray review at 12-months post-operative. If participants report the occurence of a complication at either 3-months of 12months post-operative, this is verified by a review of their hospital records.

\section{Cost effectiveness}

Health service resource data, including staff time and other resources used in the intervention, subsequent inpatient stays, outpatient and community based visits and medication are collected using hospital records and participant self-complete questionnaires. These questionnaires also collect information on social service use, travel, time off work, usual activities, informal care, and include the EQ-5D [38].

\section{Potential prognostic factors}

A number of possible potential prognostic factors are also being recorded, including socio-demographic factors, medical comorbidities, structural joint damage, health-related quality of life and psychosocial factors. The EQ-5D [38] is being used as a measure of healthrelated quality of life and the following questionnaires are being used to assess psychosocial factors: Hospital Anxiety and Depression Scale [39], Pain Self-Efficacy questionnaire [40], Illness Perceptions QuestionnaireRevised [41] and the Brief COPE [42].

\section{Nested qualitative study}

Within the RCT, there is a nested qualitative study to explore the acceptability and feasibility of the intervention for both patients and healthcare professionals. 
Interview participants are purposively sampled to include a range of socio-demographic variables so they adequately reflect those of a range of NHS patients undergoing joint replacement and NHS staff involved in joint replacement surgery. Data collection comprises of in-depth qualitative interviews with up to 25 patients at 2-3 weeks post-operative and with up to 20 healthcare professionals involved in the trial. Interviews with patients elicit experiences of surgery and post-operative recovery, acceptability of their treatment, identify information needs regarding anaesthesia and the degree to which they would like to be involved in decision-making about pain management. Interviews with healthcare professionals explore the acceptability and feasibility of the intervention and identify any potential barriers to the implementation of the intervention. The interviews are audio-recorded and fully transcribed, and themes are compared within and between one another using constant comparison techniques [43].

\section{Sample size calculation}

The primary outcome for this trial is the WOMAC Pain score at 12-months post-operative. The trial is powered to allow the THR and TKR groups to be analysed separately. Three hundred patients listed for TKR are being recruited into the study, and 150 patients will be randomised to the intervention arm and 150 to the standard care arm. The same number of patients listed for THR are being recruited and randomised. This sample size will provide $90 \%$ power to detect a difference of 0.5 standard deviations on the WOMAC Pain Scale with a two-sided 1\% significance level, and allows for a $20 \%$ dropout rate. Previous research suggests standard deviations of around $17 \mathrm{~mm}$ on the WOMAC Pain Scale before surgery [44]. Hence a difference between the treatment groups of 0.5 standard deviations equates to a difference of approximately 8-9 units on the WOMAC Pain Scale (0-100 scale).

\section{Statistical analysis}

In terms of generalisability, the characteristics of those successfully recruited into the trial will be compared with those who were eligible but declined to participate. A CONSORT diagram [45] will summarise participant flow through the study, documenting invitation and recruitment, receipt of intervention or standard care as allocated, and collection of data. To check for balance between the groups, participants in the intervention and standard care arms will be described separately in terms of demographic, stratification/minimisation variables, and baseline measures. The percentage of participants not providing primary and secondary outcome data at each of the follow-up stages will be reported for each trial arm. Factors associated with missing data (such as demographics and values of primary and important secondary outcome variables at baseline) will be explored.

Analysis will consider TKR and THR separately. The primary analysis will be implemented using a multivariable generalised linear model to investigate betweengroup differences in mean WOMAC pain score at 12 months follow-up, adjusting for minimisation variables and baseline value of the primary outcome. The type of model will depend on the distribution of the WOMAC pain score data. The primary analysis will be conducted on an intention-to-treat (ITT) basis without imputation. However, sensitivity analyses will inform the interpretation of the primary analysis. These will include an estimate of between-group difference for the primary outcome using a per-protocol analysis. In addition, the effect of missing primary outcome data will be investigated using multiple imputation methods and random effects regression models will be used to investigate any clustering of outcomes by surgeon.

The same general approach will be followed for analysis of secondary outcome measures. For additional exploratory analyses, data will be considered within each treatment group separately and so treated as two cohort studies. Analyses will examine the impact of potential risk factors, specified a priori (see section on potential prognostic factors), on pain severity at 12 -months postoperative independent of any treatment effects.

\section{Health economic analysis}

The aim of the economic evaluation is to compare from a societal perspective the costs and effects of the intervention compared to standard care. Resource use in relation to joint replacement is collected from the date of operation until the end of the trial. Health service resource use is valued using the hospital finance department data, as well as routine UK data sources. Patient and informal carer resource use is valued using selfreport and routine data sources. The analysis will consider TKR and THR participants separately. The analysis will first estimate the between group differences in mean costs using bootstrapping techniques to obtain bias corrected confidence intervals if the data are skewed. Then, incremental cost effectiveness between the two arms of the trial will be estimated using the WOMAC Pain score and the EQ-5 D to calculate Quality Adjusted Life Years (QALYs) for this population during the trial period. These results will be presented as incremental cost-effectiveness ratios and cost-effectiveness acceptability curves, derived used bootstrapping techniques. These will show the probability of the intervention being cost-effective at a range of 'willingness to pay' threshold levels.

The net monetary benefit statistic, using the difference in costs and the difference in QALYs between the two 
arms of the trial, will also be calculated for the different values of the societal willingness to pay for a year of life. In addition, because of the number of important secondary outcomes, a cost consequence analysis will be conducted in which the difference in costs and the difference in effects between the two arms of the trial are presented in tabular form. Sensitivity analysis will then be conducted to take account of uncertainty and imprecision in measurements, and will include multiple imputation models for missing values. All these analyses will be on an ITT basis and will adjust for minimisation variables.

\section{Discussion}

Many people experience chronic pain after a range of different surgical procedures $[2-4,6-8]$, and this pain is often accompanied by considerable psychological distress [46]. For patients undergoing joint replacement, chronic pain can be particularly distressing because the main reason for electing to undergo surgery is to gain relief from chronic joint pain. Therefore, for the 10-30\% [2-4] of patients who experience severe chronic pain after joint replacement, the surgery has been unsucessful in achieving its primary aim. The number of patients who develop chronic pain after joint replacement will continue to increase as the need for joint replacement increases [47], and therefore it is important that interventions to optimise outcomes after joint replacement are evaluated.

RCTs are the highest level of evidence to assess the effectivness of a surgical intervention. However, there is a paucity of well-designed and sufficiently-powered RCTs of surgical interventions, particulary within orthopaedics. Common problems with the design and reporting of orthopaedic-based RCTs include patients and outcome assessors not being blinded to treatment allocation, use of unvalidated or modified validated outcome measures, no sample size calculation being performed prior to the trial, unclear inclusion and exclusion criteria, and lack of information on the method of statistical analysis [48-51]. The APEX trial overcomes these limitations, as demonstrated by its prospective doubleblind randomised design, use of a validated primary outcome measure, sufficient statistical power to detect a clinically meaningful reduction in long-term pain severity, and clearly defined methods of analysis. In addition, the APEX trial benefits from the inclusion of a costeffectiveness analysis and a nested qualitative study to assess the acceptability and feasibility of trial participation and of the intervention.

The primary aim of the APEX trial is to determine if the addition of an intra-operative local anaesthetic wound infiltration to the standard anaesthetic regime at the Avon Orthopaedic Centre can significantly reduce the severity of joint pain at 12-months after primary THR and TKR. The results from this trial will inform evidence-based recommendations for both short-term and long-term pain management after lower limb joint replacement. If a local wound infiltration is found to be an effective and cost-effective intervention, its implementation into clinical practice could reduce the severity of chronic pain after THR and TKR, and therefore improve long-term pain outcomes for patients undergoing lower limb joint replacement.

\section{Acknowledgements and Funding}

This article outlines independent research commissioned by the National Institute for Health Research (NIHR) under its Programme Grants for Applied Research funding scheme (RP-PG-0407-10070). The views expressed are those of the author(s) and not necessarily those of the NHS, the NIHR or the Department of Health

The authors would like to acknowledge and thank Professor lan Learmonth, the RESTORE research team, the Bristol Arthroplasty Group and all staff and patients at the Avon Orthopaedic Centre who are involved in the APEX study.

The RESTORE study group consists of the following members:

Ashley Blom, Rachael Gooberman-Hill, Andrew Beswick, Vikki Wylde, Alison Smith, Emma Johnson, Jeremy Horwood, Sian Noble, Elsa Marques, Neil Artz, Luke Brunton, Victoria Wells, Sara Brookes, Samantha Dixon, Amanda Burston, lan Learmonth - University of Bristol.

Debbie Delgado, Cindy Mann, Emma Gendall, Leigh Morrison, Connie Jamera, Jenny Tyler, Dave Rea, Sophie Whitcombe, Julie Edwards, Mark Pyke, Louise Hawkins, Sue Bowman - North Bristol NHS Trust.

Paul Dieppe - Peninsula Medical School.

Cath Sackley, Paul Jepson - University of Birmingham

Pippa English-Penfold - Arthritis Care Southwest England.

\section{Author details}

${ }^{1}$ Musculoskeletal Research Unit, School of Clinical Sciences, University of Bristol, Avon Orthopaedic Centre, Southmead Hospital, Bristol, BS10 5NB, UK. ${ }^{2}$ School of Social and Community Medicine, University of Bristol, Canynge Hall, 39 Whatley Road, Bristol, BS8 2PS, UK. ${ }^{3}$ North Bristol NHS Trust, Avon Orthopaedic Centre, Southmead Hospital, Bristol, BS10 5NB, UK. ${ }^{4}$ Peninsula Medical School, Universities of Exeter and Plymouth, Plymouth, PL6 8BU, UK.

\section{Authors' contributions}

All authors conceived and designed the trial.

$\mathrm{SN}$ designed the health economic evaluation.

$\mathrm{SB}$ and $\mathrm{AS}$ designed the statistical analysis.

$\mathrm{RGH}$ and $\mathrm{JH}$ designed the nested qualitative study.

All authors drafted the manuscript.

\section{Competing interests}

The authors declare that they have no competing interests.

Received: 13 January 2011 Accepted: 26 February 2011

Published: 26 February 2011

\section{References}

1. National Joint Registry: 7th Annual Report. 2010

2. Wylde V, Dieppe P, Hewlett S, Learmonth ID: Total knee replacement: Is it really an effective procedure for all? Knee 2007, 14(6):417-423.

3. Nikolajsen L, Brandsborg B, Lucht U, Jensen TS, Kehlet H: Chronic pain following total hip arthroplasty: a nationwide questionnaire study. Acta Anaesthesio/ Scand 2006, 50(4):495-500.

4. Wylde V, Hewlett S, Learmonth I, Dieppe P: Persistent pain after joint replacement: prevalence, sensory qualities and post-operative determinants. Pain 2011, 152(3):566-572.

5. International Association for the Study of Pain: Classification of chronic pain. Descriptions of chronic pain syndromes and definitions of pain 
terms. Prepared by the International Association for the Study of Pain, Subcommittee on Taxonomy. Pain Suppl 1986, 3:S1-226.

6. Caffo O, Amichetti M, Ferro A, Lucenti A, Valduga F, Galligioni E: Pain and quality of life after surgery for breast cancer. Breast Cancer Res Treat 2003, 80(1):39-48.

7. Poobalan A, Bruce J, King P, Chambers W, Krukowski Z, Smith W: Chronic pain and quality of life following open inguinal hernia repair. British Journal of Surgery 2001, 88(8):1122-1126.

8. Perttunen K, Tasmuth T, Kalso E: Chronic pain after thoracic surgery: a follow-up study. Acta Anaesthesiologica Scandinavica 1999, 43(5):563-567.

9. Perkins FM, Kehlet $\mathrm{H}$ : Chronic pain as an outcome of surgery. A review of predictive factors. Anesthesiology 2000, 93(4):1123-1133.

10. Macrae WA: Chronic post-surgical pain: 10 years on. Br J Anaesth 2008, 101(1):77-86.

11. Dolin SJ, Cashman JN, Bland JM: Effectiveness of acute postoperative pain management: I. Evidence from published data. Br J Anaesth 2002, 89(3):409-423.

12. Wylde V, Rooker J, Halliday L, Blom A: Acute post-operative pain at rest after hip and knee arthroplasty: severity, sensory qualities and impact on sleep. Orthopaedics and Traumatology: Surgery and Research.

13. Beard DJ, Murray DW, Rees JL, Price AJ, Dodd CA: Accelerated recovery for unicompartmental knee replacement-a feasibility study. Knee 2002, 9(3):221-224.

14. Capdevila $X$, Barthelet $Y$, Biboulet $P$, Ryckwaert $Y$, Rubenovitch J, d'Athis F: Effects of perioperative analgesic technique on the surgical outcome and duration of rehabilitation after major knee surgery. Anesthesiology 1999, 91(1):8-15.

15. Jin F, Chung F: Multimodal analgesia for postoperative pain control. $J$ Clin Anesth 2001, 13(7):524-539.

16. Adam F, Chauvin M, Du Manoir B, Langlois M, Sessler DI, Fletcher D: Smalldose ketamine infusion improves postoperative analgesia and rehabilitation after total knee arthroplasty. Anesth Analg 2005, 100(2):475-480.

17. Wilmore DW, Kehlet $\mathrm{H}$ : Management of patients in fast track surgery. $B \mathrm{mj}$ 2001, 322(7284):473-476.

18. Liu SS, Richman JM, Thirlby RC, Wu CL: Efficacy of continuous wound catheters delivering local anesthetic for postoperative analgesia: a quantitative and qualitative systematic review of randomized controlled trials. J Am Coll Surg 2006, 203(6):914-932.

19. Fu P, Wu Y, Wu H, Li X, Qian Q, Zhu Y: Efficacy of intra-articular cocktail analgesic injection in total knee arthroplasty - a randomized controlled trial. Knee 2009, 16(4):280-284.

20. Bianconi M, Ferraro L, Traina GC, Zanoli G, Antonelli T, Guberti A, Ricci R, Massari L: Pharmacokinetics and efficacy of ropivacaine continuous wound instillation after joint replacement surgery. Br J Anaesth 2003, 91(6):830-835

21. Toftdahl K, Nikolajsen L, Haraldsted V, Madsen F, Tonnesen EK, Soballe K Comparison of peri- and intraarticular analgesia with femoral nerve block after total knee arthroplasty: a randomized clinical trial. Acta orthopaedica 2007, 78(2):172-179.

22. Andersen KV, Pfeiffer-Jensen M, Haraldsted V, Soballe K: Reduced hospital stay and narcotic consumption, and improved mobilization with local and intraarticular infiltration after hip arthroplasty: a randomized clinical trial of an intraarticular technique versus epidural infusion in 80 patients. Acta orthopaedica 2007, 78(2):180-186.

23. Andersen $L$, Poulsen $T$, Krogh B, Nielsen T: Postoperative analgesia in total hip arthroplasty: a randomized double-blinded, placebo-controlled study on peroperative and postoperative ropivacaine, ketorolac, and adrenaline wound infiltration. Acta orthopaedica 2007, 78(2):187-192.

24. Vendittoli PA, Makinen P, Drolet P, Lavigne M, Fallaha M, Guertin MC, Varin F: A multimodal analgesia protocol for total knee arthroplasty. A andomized, controlled study. J Bone Joint Surg Am 2006, 88(2):282-289.

25. Busch CA, Shore BJ, Bhandari R, Ganapathy S, MacDonald SJ, Bourne RB, Rorabeck CH, McCalden RW: Efficacy of periarticular multimodal drug injection in total knee arthroplasty. A randomized trial. The Journal of bone and joint surgery 2006, 88(5):959-963.

26. Gomez-Cardero P, Rodriguez-Merchan EC: Postoperative analgesia in TKA: ropivacaine continuous intraarticular infusion. Clin Orthop Relat Res 2010, 468(5):1242-1247.

27. Essving P, Axelsson K, Kjellberg J, Wallgren O, Gupta A, Lundin A: Reduced morphine consumption and pain intensity with local infiltration analgesia (LIA) following total knee arthroplasty. Acta orthopaedica 2010, 81(3):354-360

28. Chen DW, Hsieh PH, Huang KC, Hu CC, Chang YH, Lee MS: Continuous intra-articular infusion of bupivacaine for post-operative pain relief after total hip arthroplasty: a randomized, placebo-controlled, double-blind study. European journal of pain (London, England) 2010, 14(5):529-534.

29. Andersen LO, Husted H, Otte KS, Kristensen BB, Kehlet H: High-volume infiltration analgesia in total knee arthroplasty: a randomized, doubleblind, placebo-controlled trial. Acta Anaesthesiol Scand 2008, 52(10):1331-1335.

30. Parvataneni HK, Shah VP, Howard H, Cole N, Ranawat AS, Ranawat CS: Controlling pain after total hip and knee arthroplasty using a multimodal protocol with local periarticular injections: a prospective randomized study. The Journal of arthroplasty 2007, 22(6 Suppl 2):33-38.

31. Zhang J, Jiang Y, Shao JJ, Shen H, Wang Q, Zhang XL: Effect of periarticular multimodal drug injection on pain after total knee arthroplasty. [Chinese]. Journal of Clinical Rehabilitative Tissue Engineering Research 2007, 11(43):8678-8682.

32. Essving P, Axelsson K, Kjellberg J, Wallgren O, Gupta A, Lundin A: Reduced hospital stay, morphine consumption, and pain intensity with local infiltration analgesia after unicompartmental knee arthroplasty. Acta orthopaedica 2009, 80(2):213-219.

33. Vendittoli PA, Makinen P, Drolet P, Lavigne M, Fallaha M, Guertin MC, Varin F: A multimodal analgesia protocol for total knee arthroplasty. A randomized, controlled study. The Journal of bone and joint surgery American 2006, 88(2):282-289.

34. Bellamy N, Buchanan WW, Goldsmith CH, Campbell J, Stitt LW: Validation study of WOMAC: a health status instrument for measuring clinically important patient relevant outcomes to antirheumatic drug therapy in patients with osteoarthritis of the hip or knee. J Rheumatol 1988, 15(12):1833-1840.

35. Bellamy N: WOMAC: a 20-year experiential review of a patient-centered self-reported health status questionnaire. J Rheumatol 2002, 29(12):2473-2476.

36. Hawker GA, Davis AM, French MR, Cibere J, Jordan JM, March L, SuarezAlmazor M, Katz JN, Dieppe P: Development and preliminary psychometric testing of a new OA pain measure - an OARSI/OMERACT initiative. Osteoarthritis and Cartilage 2008, 16(4):409-414.

37. Freynhagen R, Baron R, Gockel U, Tolle TR: painDETECT: a new screening questionnaire to identify neuropathic components in patients with back pain. Curr Med Res Opin 2006, 22(10):1911-1920.

38. Williams A, Kind P: The present state of play about QALYs. Measure of the quality of life: the uses to which they may be put Hopkins A: RCP publications; 1992.

39. Zigmond AS, Snaith RP: The hospital anxiety and depression scale. Acta Psychiatr Scand 1983, 67(6):361-370.

40. Nicholas M: Self-efficacy and chronic pain. Paper presented at the annual conference of the British Psychological Society: 1989; St Andrews, Scotland 1989.

41. Moss-Morris R: The Revised Illness Perception Questionnaire (IPQ-R). Psychology and Health 2002, 17(1):1-16.

42. Carver CS: You want to measure coping but your protocol's too long: consider the brief COPE. Int J Behav Med 1997, 4(1):92-100.

43. Glaser B, Strauss A: The discovery of grounded theory. Chicago: Aldine; 1967.

44. Tubach F, Ravaud P, Baron G, Falissard B, Logeart I, Bellamy N, Bombardier C, Felson D, Hochberg M, van der Heijde D, et al: Evaluation of clinically relevant changes in patient reported outcomes in knee and hip osteoarthritis: the minimal clinically important improvement. Annals of the rheumatic diseases 2005, 64(1):29-33.

45. Schulz KF, Altman DG, Moher D: CONSORT 2010 statement: updated guidelines for reporting parallel group randomised trials. BMJ 2010, 340 c332.

46. Jeffrey A, Wylde V, Blom AW, Horwood J: 'Its there an I'm stuck with it': patients' experiences of chronic pain following total knee replacement surgery. Arthritis Care Res

47. Kurtz S, Ong K, Lau E, Mowat F, Halpern M: Projections of Primary and Revision Hip and Knee Arthroplasty in the United States from 2005 to 2030. The Journal of bone and joint surgery 2007, 89(4):780-785.

48. Bhandari M, Richards RR, Sprague S, Schemitsch EH: The quality of reporting of randomized trials in the Journal of Bone and Joint Surgery 
from 1988 through 2000. The Journal of bone and joint surgery 2002, 84A(3):388-396.

49. Poolman RW, Struijs PA, Krips R, Sierevelt IN, Marti RK, Farrokhyar F, Bhandari M: Reporting of outcomes in orthopaedic randomized trials: does blinding of outcome assessors matter? The Journal of bone and joint surgery 2007, 89(3):550-558.

50. Freedman KB, Back S, Bernstein J: Sample size and statistical power of randomised, controlled trials in orthopaedics. The Journal of bone and joint surgery 2001, 83(3):397-402.

51. Chan S, Bhandari M: The quality of reporting of orthopaedic randomized trials with use of a checklist for nonpharmacological therapies. The Journal of bone and joint surgery 2007, 89(9):1970-1978.

\section{Pre-publication history}

The pre-publication history for this paper can be accessed here: http://www.biomedcentral.com/1471-2474/12/53/prepub

doi:10.1186/1471-2474-12-53

Cite this article as: Wylde et al.: The effect of local anaesthetic wound infiltration on chronic pain after lower limb joint replacement: A protocol for a double-blind randomised controlled trial. BMC

Musculoskeletal Disorders 2011 12:53.

\section{Submit your next manuscript to BioMed Central} and take full advantage of:

- Convenient online submission

- Thorough peer review

- No space constraints or color figure charges

- Immediate publication on acceptance

- Inclusion in PubMed, CAS, Scopus and Google Scholar

- Research which is freely available for redistribution

Submit your manuscript at www.biomedcentral.com/submit 